OPEN Microstructural Abnormalities in the

SUBJECT AREAS:

DEVELOPMENTAL

DISORDERS

MAGNETIC RESONANCE IMAGING

Received

10 June 2014

Accepted

14 October 2014

Published

3 November 2014

Correspondence and requests for materials should be addressed to X.X.D. (merryxiaoxia@

163.com) or Q.Y.G. (qiyonggong@hmrrc. org.cn)

* These authors contributed equally to this work.

\title{
Combined and Inattentive Subtypes of Attention Deficit Hyperactivity Disorder: a Diffusion Tensor Imaging Study
}

\author{
Du Lei ${ }^{1,3 *}$, Jun $\mathrm{Ma}^{2 *}$, Xiaoxia Du³, Guohua Shen ${ }^{3}$, Xingming Jin² \& Qiyong Gong'
}

\begin{abstract}
'Huaxi MR Research Center (HMRRC), Department of Radiology, West China Hospital of Sichuan University, Chengdu, Sichuan 610041, China, ${ }^{2}$ Shanghai Key Laboratory of Children's Environmental Health, Department of Developmental and Behavioral Pediatrics of Shanghai Children's Medical Center, Xinhua Hospital Affiliated to Shanghai Jiao Tong University School of Medicine, Shanghai 200062, China, ${ }^{3}$ Shanghai Key Laboratory of Magnetic Resonance, Department of Physics, East China Normal University, Shanghai 200062, China.
\end{abstract}

Previous research has demonstrated that there are specific white matter abnormalities in patients with attention deficit/hyperactivity disorder (ADHD). However, the results of these studies are not consistent, and one of the most important factors that affects the inconsistency of previous studies maybe the ADHD subtype. Different ADHD subtypes may have some overlapping microstructural damage, but they may also have unique microstructural abnormalities. The objective of this study was to investigate the microstructural abnormalities associated with two subtypes of ADHD: combined (ADHD-C) and inattentive (ADHD-I). Twenty-eight children with ADHD-C, 28 children with ADHD-I and 28 healthy children participated in this study. Fractional anisotropy (FA), radial diffusivity (RD) and axial diffusivity (AD) were used to analyze diffusion tensor imaging (DTI) data to provide specific information regarding abnormal brain areas. Our results demonstrated that ADHD-I is related to abnormalities in the temporo-occipital areas, while the combined subtype (ADHD-C) is related to abnormalities in the frontal-subcortical circuit, the fronto-limbic pathway, and the temporo-occipital areas. Moreover, an abnormality in the motor circuit may represent the main difference between the ADHD-I and ADHD-C subtypes.

\footnotetext{
$\Lambda$ ttention deficit/hyperactivity disorder (ADHD) is one of the most common childhood neurodevelopmental disorders, which affects approximately $3-7 \%$ of school-age children ${ }^{1}$ and is characterized by ageinappropriate symptoms of inattention, hyperactivity, impulsivity and motor restlessness. According to the ADHD criteria listed in the Diagnostic and Statistical Manual of Mental Disorders, 4th Edition (DSM-IV), the core symptoms of ADHD include inattention, impulsiveness and hyperactivity. There are three defined subtypes of ADHD: predominantly hyperactive-impulsive (ADHD-H), predominantly inattentive (ADHD-I), and combined (ADHD-C). ADHD-I and ADHD-C are the main subtypes of ADHD, and ADHD-H is rarely identified clinically ${ }^{2}$. Previous research has implicated a combination of environmental, genetic and biological factors in $\mathrm{ADHD}^{3,4}$. However, the etiology and pathophysiology of ADHD are not completely understood, particularly concerning subtype differences.

Diffusion tensor imaging (DTI) is an magnetic resonance imaging (MRI) technique that has increasingly been used to characterize the microstructure of white matter by exploiting the diffusion characteristics of water molecules in the brain ${ }^{5}$. One DTI measure is fractional anisotropy (FA), which quantifies the directionality of diffusion. FA is the most commonly used parameter for the investigation of anisotropy, which is a fairly nonspecific biomarker of microstructural architecture and neuropathology ${ }^{6}$. Greater specificity regarding the neurobiological determinates of the altered white matter structure may be achieved via the examination of axial diffusivity $(\mathrm{AD})$ and radial diffusivity $(\mathrm{RD})$, which provide insights into the underlying mechanism of FA changes. $\mathrm{AD}$, which measures diffusion parallel to the axonal fibers, has been correlated with axonal injury ${ }^{7}$ and/or axonal pruning ${ }^{8}$. RD measures diffusion perpendicular to the direction of fibers, which may be related to myelin injury and/or decreased myelination ${ }^{9,10}$.
} 
In recent years, there has been an increased use of neuroimaging to investigate ADHD. These neuroimaging studies suggest that abnormalities in brain structure and function may play important roles in the pathophysiology of $\mathrm{ADHD}^{11-14}$. However, a limited number of studies have used the DTI technique on patients with ADHD, and their results are not consistent. For example, most previous studies have reported decreased FA in ADHD patients ${ }^{15-17}$, whereas others studies have observed increased FA in ADHD patients ${ }^{18,19}$. Although a recent meta-analysis of DTI studies on $\mathrm{ADHD}^{14}$ attempted to identify consistent results across studies, it is difficult to draw firm conclusions because there are many factors that may affect the results, including the participants' ages, statistical thresholds, and MRI scanners employed. Among these factors, one of the most important factors that affects the consistency of previous studies maybe the ADHD subtype. First, ADHD patients exhibit different symptoms and can be diagnosed and classified into different subtypes. Second, previous studies have suggested that different ADHD subtypes constitute neurobiologically and behaviorally distinct conditions ${ }^{20,21}$.

According to the DSM-IV, ADHD-C is characterized with symptoms of both inattention and hyperactivity/impulsivity. ADHD-I shares inattentive symptoms with the combined subtype, but it lacks of hyperactive and impulsive behaviors. Solanto et al. compared the social functioning of children with ADHD-C and those with ADHD$\mathrm{I}$, and it was observed that children with ADHD-I were impaired in assertiveness, whereas children with ADHD-C shown deficit in selfcontrol $^{22}$. Furthermore, ADHD-C and ADHD-I were reported to be distinguishable in their comorbidities and their behavioral, emotional, social, academic, and cognitive functioning ${ }^{23}$. However, imaging studies have generally ignored these distinctions thus lead to inconstant results. We thus hypothesize that $\mathrm{ADHD}-\mathrm{C}$ and ADHD-I have differentially microstructural changes in the brain areas related to attention and execution function in patients with ADHD.

The purpose of this study was therefore to examine the differences in brain microstructure between healthy children and children with ADHD-I and ADHD-C. In this study, we subtyped our subjects based on their specific type of ADHD and matched the samples to the healthy controls. Moreover, the combination of FA, AD, and RD analyses employed in present study would provide detailed and specific information for our understanding of the underlying neuropsychopathological mechanism of the ADHD.

\section{Results}

Demographic and clinical comparisons. Demographics and clinical characteristics of the participants are presented in Table 1. There were no significant differences in the age, gender distribution, or duration of school education between the ADHD subjects and healthy controls.
Comparison of the ADHD-I and control groups. The children with ADHD-I exhibited significant changes in the left occipital lobe/ cuneus (increased RD), the left middle temporal gyrus (decreased $\mathrm{AD}$ ), and the left superior temporal gyrus (increased RD). The detailed results are shown in Figure 1 and Table 2.

Comparison of the ADHD-C and control groups. Compared with the healthy children, the children with ADHD-C demonstrated significantly decreased $\mathrm{AD}$ and $\mathrm{RD}$ in the left middle frontal gyrus, the supplementary motor area, and the left precuneus. FA decreased in the left parahippocampal gyrus, and $\mathrm{AD}$ decreased in the left precuneus and the right cingulate cortex. Additionally, increased $\mathrm{RD}$ was identified in the following brain areas: the right fusiform gyrus, the left cuneus, the left lingual gyrus, the left superior temporal gyrus and the right middle temporal gyrus. The detailed results are shown in Figure 1 and Table 2.

Comparison of the ADHD-C and ADHD-I groups. Several clusters exhibited significant differences between the ADHD-C and ADHD-I groups. We identified significant differences in the following brain areas: the right thalamus (increased FA and RD), the left postcentral gyrus (increased $\mathrm{AD}$ and $\mathrm{RD}$ ), the supplementary motor area (SMA) (increased $\mathrm{RD}$ ), and the right caudate (increased $\mathrm{AD})$. The detailed results are shown in Figure 2 and Table 3.

\section{Discussion}

To our knowledge, this is the first study to use DTI to investigate the differences in neural structures between ADHD subtypes. We explored microstructure abnormalities in children between 7-13 years of age who exhibited the combined or inattentive subtypes of ADHD using both the FA value and specialized DTI eigenvalues (AD and $\mathrm{RD}$ ).

Microstructural changes in ADHD-I. The children with ADHD-I exhibited abnormalities in the left occipital lobe/cuneus (increased $\mathrm{RD}$ ), the left middle temporal gyrus (decreased AD), and the left superior temporal gyrus (increased RD).

The cuneus has been reported to be affected by ADHD and is involved in vision and the default mode network ${ }^{24}$. A previous study has suggested that adults with ADHD display a significant increase in connectivity in the left cuneus during working memory processing compared with healthy controls ${ }^{25}$. Another study also reported that ADHD patients have altered regional homogeneity during the resting state in the bilateral cuneus and the precuneus, as well as a significant positive correlation with inattentive scores ${ }^{26}$. Thus, our findings may suggest that abnormalities in the cuneus play a role in the pathology of ADHD and may be related to inattentive symptoms.

The middle and superior temporal gyri have been reported to be abnormal in ADHD. One study demonstrated that there were delays in cortical maturation in the temporal cortex of $\mathrm{ADHD}$ patients,

Table 1 | Demographics and clinical characteristics of the participants

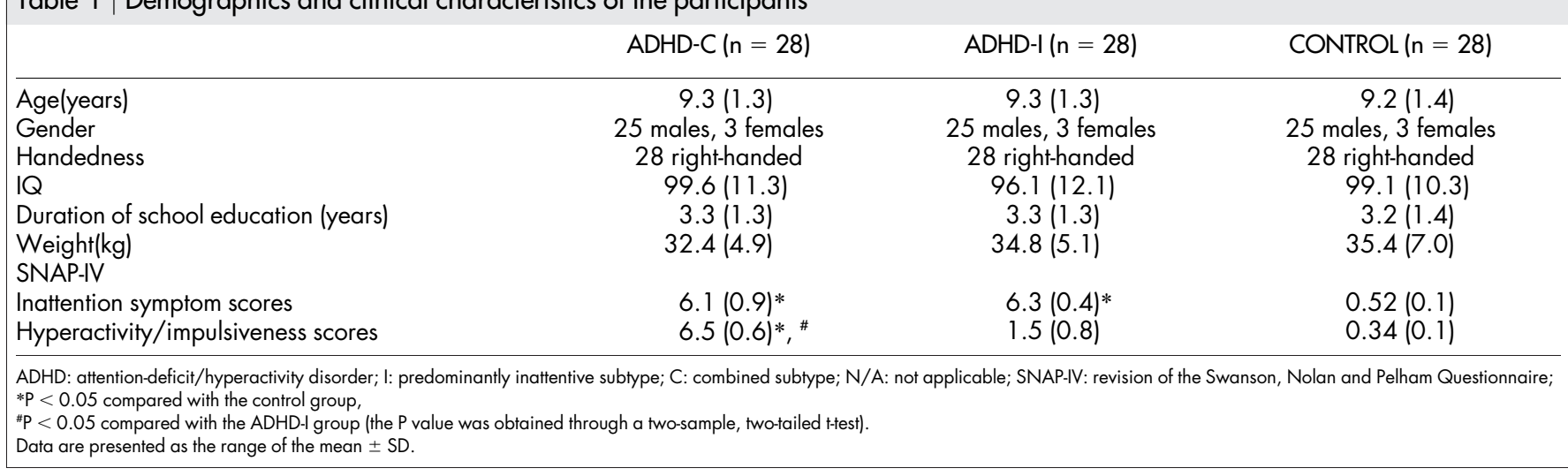


A
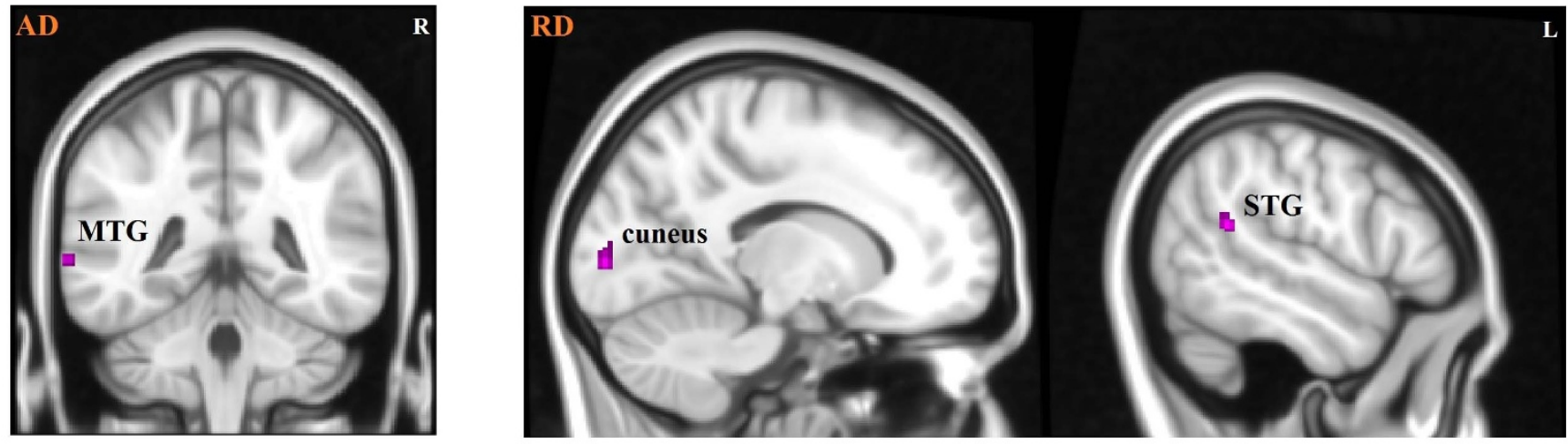

B
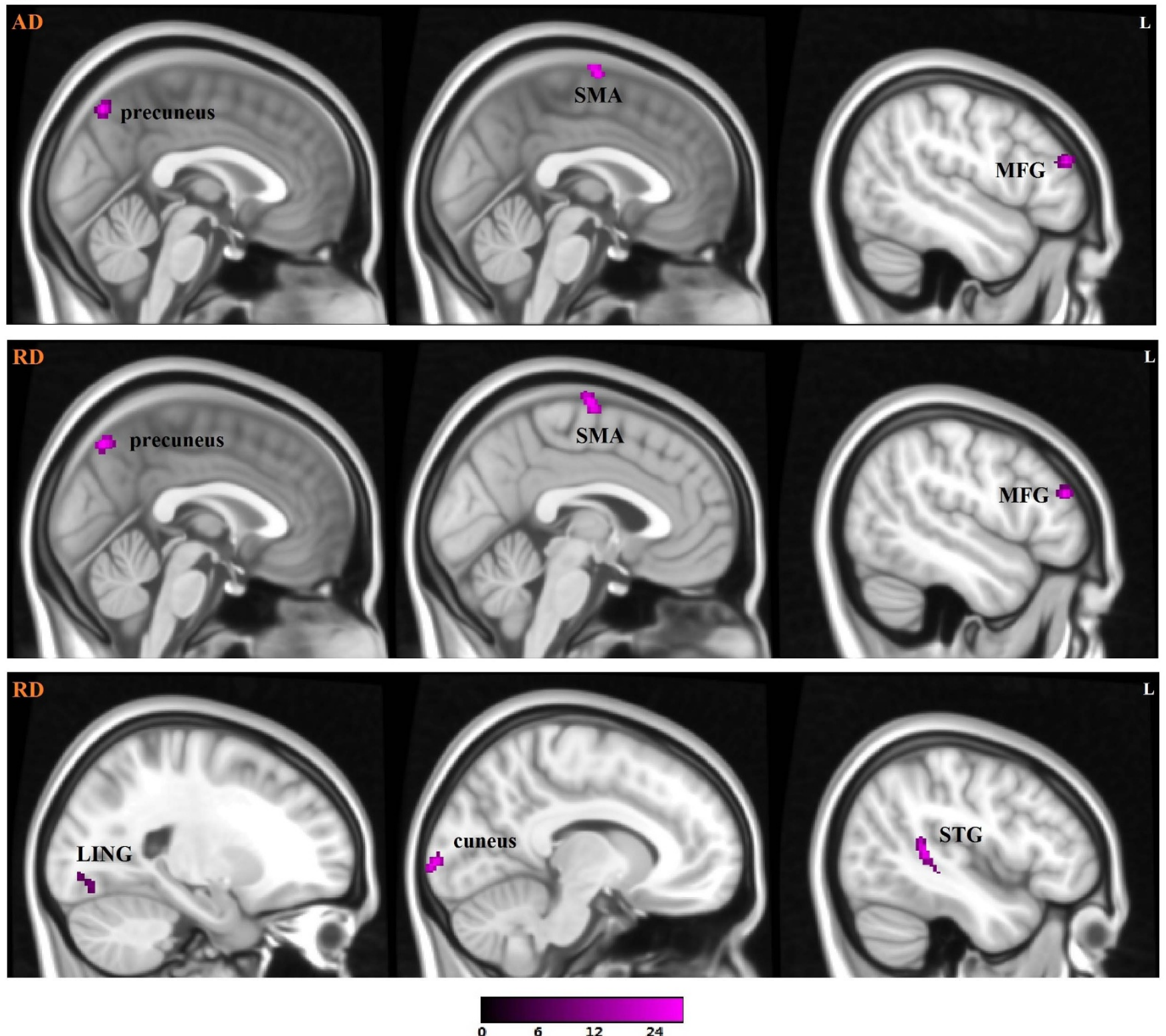

Figure $1 \mid$ (A) Compared with healthy children, children with ADHD-I exhibited significant changes in diffusion parameters. (B) Compared with healthy children, children with ADHD-C exhibited significant changes in diffusion parameters. Abbreviations: MTG, middle temporal gyrus; STG, superior temporal gyrus; SMA, supplementary motor area; MFG, middle frontal gyrus; LING, Lingual gyrus. 


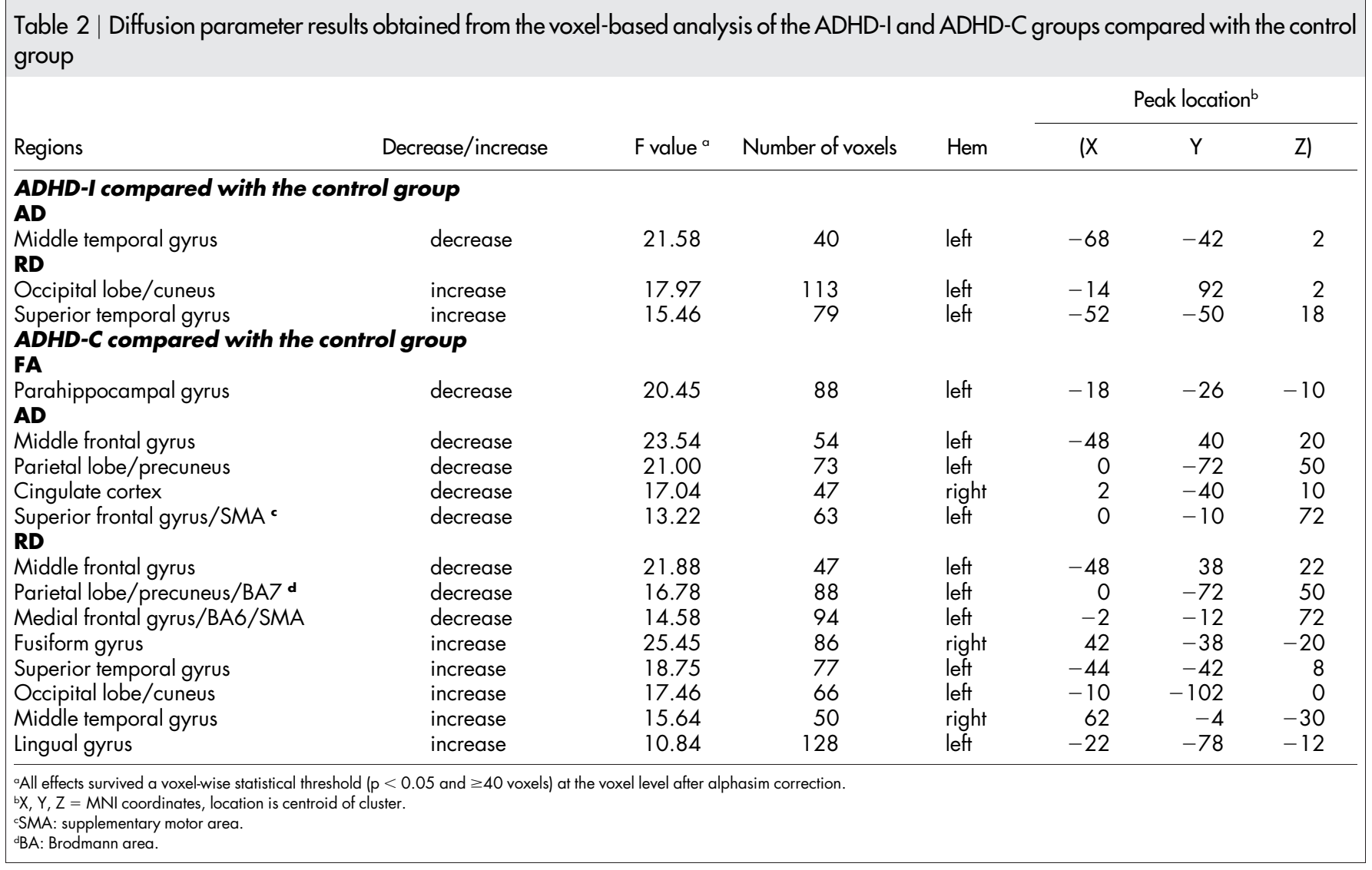

which occurred bilaterally in the middle/superior temporal gyrus ${ }^{27}$. Sowell et al. observed the reduction in size of the anterior temporal lobes along with an increased density of grey matter in more posterior temporal cortices ${ }^{28}$. Moreover, a study that used both structural and functional MRI demonstrated that the differences between patients with ADHD and healthy controls were largely located in the temporal lobe ${ }^{29}$. An fMRI study demonstrated that children with ADHD revealed a relative increase in the activation of the middle/ inferior temporal cortex during a go/no-go task ${ }^{30}$. Dibbets et al. also reported that adults with $\mathrm{ADHD}$ engaged the middle temporal gyrus
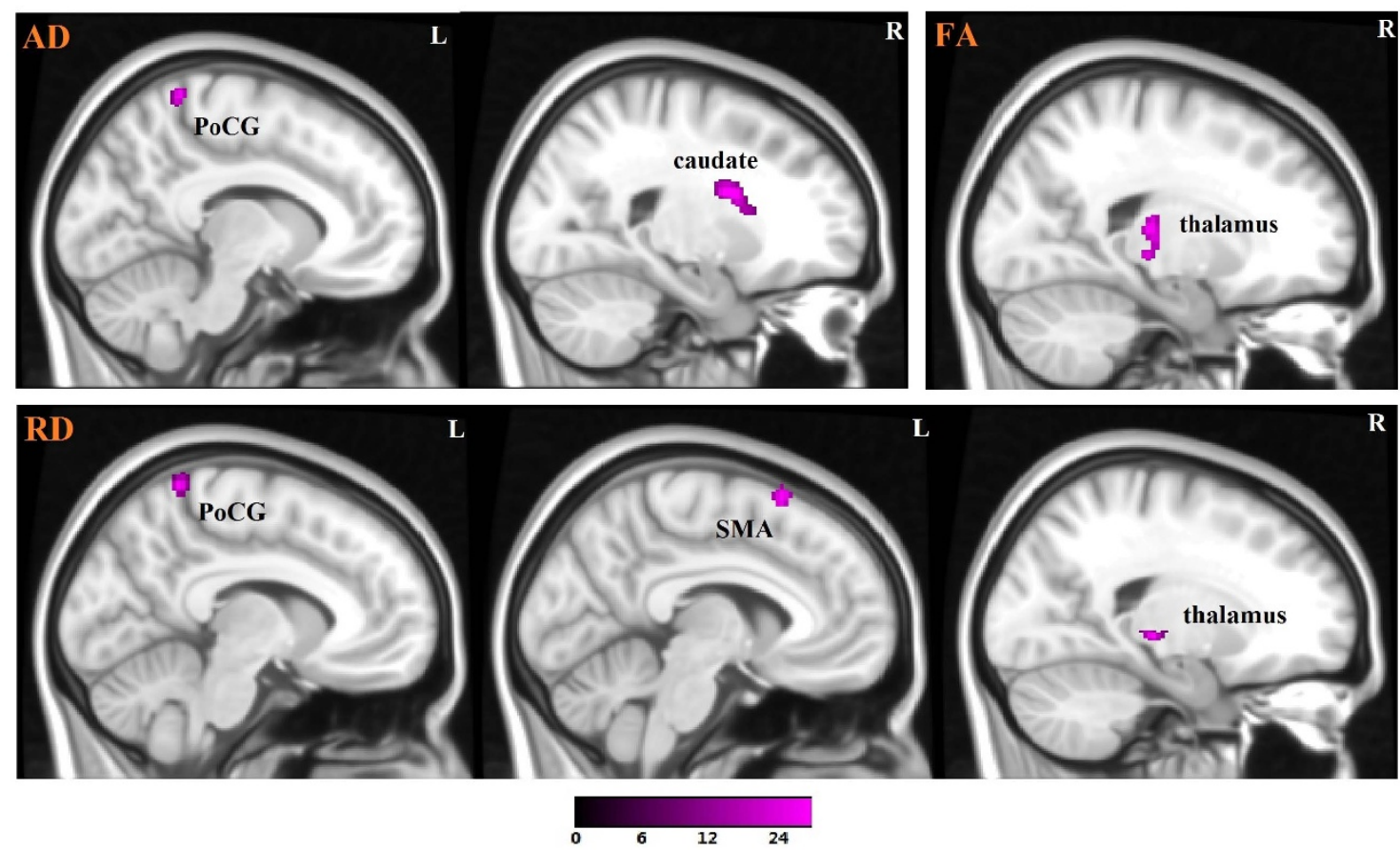

Figure $2 \mid$ Compared with children with ADHD-I, children with ADHD-C exhibited significant changes in diffusion parameters. Abbreviations: SMA, supplementary motor area; PoCG, postcentral gyrus. 


\begin{tabular}{|c|c|c|c|c|c|c|c|}
\hline \multirow[b]{2}{*}{ Regions } & \multirow[b]{2}{*}{ Decrease/increase } & \multirow[b]{2}{*}{$F_{\text {value }}{ }^{a}$} & \multirow[b]{2}{*}{ Number of voxels } & \multirow[b]{2}{*}{ Hem } & \multicolumn{3}{|c|}{ Peak location ${ }^{b}$} \\
\hline & & & & & (X & Y & Z) \\
\hline \multicolumn{8}{|l|}{ FA } \\
\hline \multicolumn{7}{|l|}{ AD } & 10 \\
\hline Caudate & increase & 16.49 & 103 & right & 22 & 6 & 22 \\
\hline Postcentral gyrus/BA5 c & increase & 20.61 & 76 & left & -8 & -50 & 70 \\
\hline Thalamus & increase & 17.34 & 70 & right & 20 & -24 & -2 \\
\hline Superior frontal gyrus/BA6/SMA d & increase & 13.19 & 49 & left & -6 & 18 & 64 \\
\hline
\end{tabular}

more during task switching compared with healthy controls ${ }^{31}$. These temporal regions integrate information from lower-order sensory to higher-order perceptual functions, and guide the control of attention and action ${ }^{27}$. Thus, our results suggest that the observed abnormalities in the left middle and superior temporal gyri are related to the developmental delay or abnormality, which in turn, affects the control of attention and action. In summary, we demonstrated ADHD-I patients exhibited abnormalities in the temporo-occipital areas, which may affect visual information processing and integration and subsequently the control of attention and action.

Microstructural changes in ADHD-C. Compared with the healthy children, the children with ADHD-C exhibited abnormalities in the temporal lobe (the left superior temporal gyrus, the right middle temporal gyrus and the right fusiform gyrus) and the occipital lobe (the cuneus and the lingual gyrus), which was similar to ADHD-I. These results suggest that the temporo-occipital areas are abnormal in patients either with the ADHD-I or with ADHD-C. In addition, we also identified abnormalities in the precuneus in the children with ADHD-C, and this is consistent with a previous fMRI study that decreased activation was observed in the right parieto-occipital areas (including the cuneus and the precuneus, BA 19) ${ }^{32}$. This result was thought to be associated with reduced mental rotation/ spatial working memory in children with the ADHD-C subtype ${ }^{32}$.

The children with ADHD-C also exhibited significant microstructural abnormalities in the frontal lobe, including the middle frontal gyrus and the SMA. These results may suggest that frontal-subcortical circuits, including both motor circuits (that originate in the SMA) and behaviorally relevant circuits (that originate in the prefrontal cortex $)^{33}$, are abnormal in children with the ADHD-C subtype. Additionally, the children with ADHD-C exhibited abnormalities in the limbic lobe, specifically in the left parahippocampal gyrus and the right cingulate cortex. Previous DTI studies have revealed microstructural abnormalities in the prefrontal cortex ${ }^{16,18}$ and the fronto-limbic regions ${ }^{34}$ of ADHD patients. Consistent with previous studies, our results may also suggest abnormalities in the fronto-limbic pathway in patients with the ADHD-C subtype.

Significant difference in the microstructural changes in ADHD-C and ADHD-I. When we directly compared the ADHD-C and ADHD-I groups, there were many regional differences observed, primarily in the left postcentral gyrus, the right thalamus, the left SMA and the right caudate, all of which have been reported to be abnormal in ADHD patients ${ }^{13,35-38}$. Decreased caudate volumes in ADHD patients have been well documented ${ }^{13}$, and there is study reported the consistent decreased volume of the right caudate nucleus in ADHD samples ${ }^{35}$. Xia et al. identified significant regional atrophy in the left thalamus in children with ADHD compared with controls, which suggested that the structural abnormalities within the thalamus and adjacent brain regions may account for the impaired cognitive performances in the attention and executive function domains in $\mathrm{ADHD}^{36}$. Compared with controls, adults with ADHD exhibited an increased thickness in the primary somatosensory cortex (postcentral gyrus) ${ }^{37}$ in addition to the altered resting state connectivity of the supplementary motor area in the ventral attention network ${ }^{38}$. Moreover, the SMA, the postcentral gyrus (i.e., the primary somatosensory cortex), the caudate nucleus (part of the striatum that belongs to the basal ganglia) and the thalamus are all involved in the motor pathway ${ }^{39}$. The frontostriatal and fronto-subthalamic circuits (i.e., the inferior frontal cortex, the SMA, the caudate nucleus and the thalamus) are thought to involve response inhibition ${ }^{33,40,41}$. Furthermore, consistent with our hypothesis, the ADHD-C and ADHD-I subtypes have differences in the motor circuit. ADHD appears to be related to deficit in response inhibition ${ }^{42,43}$. Thus, the frontalsubcortical circuits involved in motor control and inhibition may be related to the impulsiveness and hyperactivity in children with ADHD-C ${ }^{33}$. Our results indicate that abnormalities in motor circuits may represent the main difference between ADHD-I and ADHD-C.

In most previous studies, the ADHD patient group has comprised patients with different ADHD subtypes; thus, the obtained results may be a result of the different proportions of the subtypes. Consistent with our hypothesis, our results demonstrate that there are significant differences between ADHD-C and ADHD-I patients and that ADHD-C patients exhibit more serious and significant microstructural changes, which is consistent with their ADHD symptoms: ADHD-C patients present more serious behavioral problems compared with ADHD-I patients. Thus, we suggest that future studies regarding ADHD should account for the different disease subtypes.

Combination of FA, RD and AD. We investigated the white matter abnormalities in ADHD with the use of both FA and specific DTI eigenvalues, i.e., $\mathrm{AD}$ and $\mathrm{RD}$. Animal studies suggest that dysmyelination lead to an increase in the RD measure, without significantly changing $\mathrm{AD}$, whereas axonal damage leads to a decrease in $\mathrm{AD}^{9,44}$. Reductions in $\mathrm{RD}$ are thought to result from a lower degree of neuronal branching ${ }^{45}$. Analyses of eigenvalues based on $\mathrm{AD}$ and $\mathrm{RD}$ are likely to provide us with further insights into the underlying neurobiological mechanisms of the disease.

According to the observed changes of AD and RD in ADHD-I, we speculate that there are myelination deficits in the left occipital lobe/ cuneus and the left superior temporal gyrus ${ }^{9}$, in addition to axonal damage or degeneration in the left middle temporal gyrus ${ }^{7,44}$. 
Furthermore, ADHD-C may have dysmyelination in the right fusiform gyrus, the left cuneus, the left lingual gyrus, the left superior temporal gyrus and the right middle temporal gyrus ${ }^{9}$, and the axonal damage or degeneration in the left middle frontal gyrus, the left precuneus, the right cingulate cortex and the left $\mathrm{SMA}^{7,44}$. Additionally, ADHD-C also have a lower degree of neuronal branching in the left middle frontal gyrus, the left precuneus and the left $\mathrm{SMA}^{45}$.

Several issues must be considered here. First, because ADHD-H is rarely observed clinically, our study included only two ADHD subtypes: ADHD-I and ADHD-C. Future studies that include the third subtype (ADHD-H) would provide more comprehensive insights into the psychopathology of ADHD. Second, we excluded children with motor deficits or motor developmental delays in this study. The presence of motor deficits in children with ADHD should be assessed or identified in detail in future studies. Third, a technical limitation of this work is that the gradient directions of our DTI data are relatively minimal. Future studies should use additional gradient directions during MRI acquisition so to improve the accuracy of fiber tractography, along with the exploration of neurocircuitry in ADHD. Finally, although the DSM-5 "presentations" have somewhat reduced the relevance of the DSM-IV ADHD subtypes, the assessment of the different neurobiological underpinnings of predominantly inattentive vs. combined ADHD symptoms is still of major interest.

Conclusion. In summary, our whole-brain-based analysis demonstrated that ADHD-I and ADHD-C patients display different microstructural changes in their brains, which suggests that the ADHD inattention subtypes related to abnormalities in the temporo-occipital areas and the combined subtype is related to abnormalities in the frontal-subcortical circuits, the fronto-limbic pathway, and the temporo-occipital areas. Moreover, the result from our comparison of ADHD-I and ADHD-C subgroups indicated that the abnormalities in the motor circuits may represent the main difference between these two subtypes.

\section{Methods}

Participants. Seventy-eight children with ADHD and 58 healthy children between the ages of 7 and 13 years participated in the study. All 78 children with ADHD were outpatients at Shanghai Children's Medical Center. The ADHD patients had not previously used psychiatric medication. The study protocol was designed in accordance with the guidelines outlined in the Declaration of Helsinki and was approved by the Intramural Research Board of Shanghai Children's Medical Center (No: SCMC-201014). All children and their guardians provided written informed consent prior to participation in the study.

The inclusion criteria for all participants were as follows: (1) a physical, psychiatric, and neurological evaluation was conducted by at least 3 members of a team of certified and experienced developmental and behavioral pediatricians; (2) age 7-13 years; (3) right-handedness; (4) all children were diagnosed with ADHD based on the DSM-IV criteria (American Psychiatric Association, Diagnostic and Statistical Manual of Mental Disorders, 4th Edition); (5) a SNAP-IV (revision of the Swanson, Nolan and Pelham questionnaire $)^{46}$ evaluation was performed to scale the ADHD symptom scores $^{47}$; (6) the Wechsler Intelligence Scale for Children-Revised (WISC-R) test was employed to determine the intelligence quotient (IQ) of all subjects; and (7) the number, age, and gender of each subgroup were matched.

The exclusion criteria for this study were as follows: (1) conduct disorder, oppositional defiant disorder, Tourette syndrome, or any other axis I psychiatric comorbid disorders; (2) previous head trauma, neurologic disorders, psychosurgery, or substantial physical illness; (3) motor deficits or motor developmental delay; (4) history of stimulant or other medication to treat inattention problems;(5) DTI data with obvious artifacts and distortions; (6) left-handedness, as assessed with the Annett Hand Preference Questionnaire; and (7)a full-scale IQ less than 80 according to an age appropriate WISC- Chinese Revision.

Finally, 56 children with ADHD and 28 healthy children matched for age, sex, and exclusion criteria (3) and (4) were included in our study. The children were placed into one of three groups: an ADHD-C group, an ADHD-I group or children with normal development (control group). There were 3 females and 25 males in each group. The children with ADHD-I had a mean age of 9.3 years $(\mathrm{SD}=1.3)$, while the children with ADHD-C had a mean age of 9.3 years $(S D=1.3)$. The healthy controls had a mean age of 9.2 years $(\mathrm{SD}=1.4)$.
Data acquisition. The MRI data were acquired on a Siemens 3T Trio MR scanner with a 12-channel phased array coil. DTI acquisition involved a single-shot, spin-echo planar imaging sequence in contiguous axial planes that covered the whole brain. Diffusion-sensitizing gradients were applied in 12 non-collinear directions, together with acquisition without diffusion weighting $(b=0)$. The imaging parameters were set to the following values: $\mathrm{TR}=6,600 \mathrm{~ms}, \mathrm{TE}=89 \mathrm{~ms}$, average $=4$, b-value $=$ $1,000 \mathrm{~s} / \mathrm{mm}^{2}$, slice thickness $=2.5 \mathrm{~mm}, 50$ slices. The matrix resolution was acquired at $128 \times 128$ and reconstructed to $256 \times 256$. The resolution was $2 \times 2 \times 2.5 \mathrm{~mm}^{3}$. The subjects were told not to move during the scans, and a Siemens dedicated filler was used to prevent head movement. The acquisition time for the scan was 6 minutes and 5 seconds.

Data processing and statistical analysis. SPM 8 (http://www.fil.ion.ucl.ac.uk/spm/), MATLAB 2010 (MathWorks, Natick, MA) and FSL4.1 (http://www.fmrib.ox.ac.uk/ $\mathrm{fsl} /$ ) were used to analyze the data. First, the DICOM files of each DTI acquisition were converted into a single multivolume NIFTI file. Then, FSL's "eddy current correction" was used to correct the distortions induced by the eddy current and head motion in the dataset. The brain was extracted for further processing using BET (Brain Extraction Tool, http://fsl.fmrib.ox.ac.uk/fsl/bet2/). Finally, the FA, RD and AD maps were calculated with FSL.

The correction of head motion image artifacts, normalization and statistical analyses were performed using SPM 8. To ensure that our data were accurately matched, we developed acustomized pediatric template using SPM to reduce potential errors caused by matching to an adult template ${ }^{48}$. The procedure for the development of the optimized template consisted of three steps. First, we normalized the FA maps of the control group based on the deformation information generated from the corresponding unweighted images (first $\mathrm{b}=0$ image) and the echo-planar imaging (EPI) template (in MNI152 space). These maps were termed wFA maps. The wFA maps were averaged to produce a mean map. Finally, the mean map was smoothed (using a $[6,6,6]$ FWHM Gaussian kernel) to obtain our customized pediatric template, which was used for further analysis ${ }^{49}$.

We normalized all original FA maps (from both the patient and control groups) to the deformation field produced from the original FA maps and our FA-specific pediatric template. The normalized FA maps were smoothed using a $[6,6,6]$ filter for statistical analysis. Finally, a fractional design specification was employed to compare the ADHD-C, ADHD-I and control groups via one-way ANOVA. An intergroup three-sample F-test comparison was performed using an absolute threshold of FA $>$ 0.15 . The same processes were applied to the $\mathrm{AD}$ and $\mathrm{RD}$ maps. For all analyses, the statistical map cluster level had a threshold of $\mathrm{p}<0.005$ (Alphasim corrected, $\mathrm{p}<$ 0.05 and voxels $\geq 40$ ). This correction was determined through Monte Carlo simulations ${ }^{50}$ using AFNI AlphaSim (http://afni.nimh.nih.gov/afni/).

1. Polanczyk, G., de Lima, M. S., Horta, B. L., Biederman, J. \& Rohde, L. A. The worldwide prevalence of ADHD: a systematic review and metaregression analysis. Am J Psychiatry 164, 942-948 (2007).

2. Skounti, M. et al. Prevalence of attention deficit hyperactivity disorder in schoolchildren in Athens, Greece. Association of ADHD subtypes with social and academic impairment. Atten Defic Hyperact Disord 2, 127-132 (2010).

3. Curatolo, P., D'Agati, E. \& Moavero, R. The neurobiological basis of ADHD. Ital J Pediatr 36, 79 (2010).

4. Tripp, G. \& Wickens, J. R. Neurobiology of ADHD. Neuropharmacology 57, 579-589 (2009).

5. Beaulieu, C. The basis of anisotropic water diffusion in the nervous system - a technical review. NMR Biomed 15, 435-455 (2002).

6. Alexander, A. L., Lee, J. E., Lazar, M. \& Field, A. S. Diffusion tensor imaging of the brain. Neurotherapeutics 4, 316-329 (2007).

7. Budde, M. D., Xie, M., Cross, A. H. \& Song, S. K. Axial diffusivity is the primary correlate of axonal injury in the experimental autoimmune encephalomyelitis spinal cord: a quantitative pixelwise analysis. J Neurosci 29, 2805-2813 (2009).

8. Bockhorst, K. H. et al. Early postnatal development of rat brain: in vivo diffusion tensor imaging. J Neurosci Res 86, 1520-1528 (2008).

9. Song, S. K. et al. Dysmyelination revealed through MRI as increased radial (but unchanged axial) diffusion of water. Neuroimage 17, 1429-1436 (2002).

10. Song, S. K. et al. Diffusion tensor imaging detects and differentiates axon and myelin degeneration in mouse optic nerve after retinal ischemia. Neuroimage 20, 1714-1722 (2003).

11. Cubillo, A., Halari, R., Smith, A., Taylor, E. \& Rubia, K. A review of fronto-striatal and fronto-cortical brain abnormalities in children and adults with Attention Deficit Hyperactivity Disorder (ADHD) and new evidence for dysfunction in adults with ADHD during motivation and attention. Cortex 48, 194-215 (2012).

12. Pastura, G., Mattos, P., Gasparetto, E. L. \& Araujo, A. P. Advanced techniques in magnetic resonance imaging of the brain in children with ADHD. Arq Neuropsiquiatr 69, 242-252 (2011).

13. Frodl, T. \& Skokauskas, N. Meta-analysis of structural MRI studies in children and adults with attention deficit hyperactivity disorder indicates treatment effects. Acta Psychiatr Scand 125, 114-126 (2012).

14. van Ewijk, H., Heslenfeld, D. J., Zwiers, M. P., Buitelaar, J. K. \& Oosterlaan, J. Diffusion tensor imaging in attention deficit/hyperactivity disorder: A systematic review and meta-analysis. Neurosci Biobehav Rev 36, 1093-1106 (2012).

15. Ashtari, M. et al. Attention-deficit/hyperactivity disorder: a preliminary diffusion tensor imaging study. Biol Psychiatry 57, 448-455 (2005). 
16. Konrad, A. et al. Disturbed structural connectivity is related to inattention and impulsivity in adult attention deficit hyperactivity disorder. Eur J Neurosci 31, 912-919 (2010).

17. Konrad, A. et al. White matter abnormalities and their impact on attentional performance in adult attention-deficit/hyperactivity disorder. Eur Arch Psychiatry Clin Neurosci 262, 351-360 (2012).

18. Davenport, N. D., Karatekin, C., White, T. \& Lim, K. O. Differential fractional anisotropy abnormalities in adolescents with ADHD or schizophrenia. Psychiatry Res 181, 193-198 (2010).

19. Li, Q. et al. Increased fractional anisotropy in white matter of the right frontal region in children with attention-deficit/hyperactivity disorder: a diffusion tensor imaging study. Neuro Endocrinol Lett 31, 747-753 (2010).

20. Diamond, A. Attention-deficit disorder (attention-deficit/hyperactivity disorder without hyperactivity): a neurobiologically and behaviorally distinct disorder from attention-deficit/hyperactivity disorder (with hyperactivity). Dev Psychopathol 17, 807-825 (2005).

21. Carr, L., Henderson, J. \& Nigg, J. T. Cognitive control and attentional selection in adolescents with ADHD versus ADD. J Clin Child Adolesc Psychol 39, 726-740 (2010)

22. Solanto, M. V., Pope-Boyd, S. A., Tryon, W. W. \& Stepak, B. Social functioning in predominantly inattentive and combined subtypes of children with ADHD. J Atten Disord 13, 27-35 (2009).

23. Baeyens, D., Roeyers, H. \& Walle, J. V. Subtypes of attention-deficit/hyperactivity disorder (ADHD): distinct or related disorders across measurement levels? Child Psychiatry Hum Dev 36, 403-417 (2006).

24. Alonso Bde, C. et al. A multi-methodological MR resting state network analysis to assess the changes in brain physiology of children with ADHD. PLoS One 9, e99119 (2014).

25. Wolf, R. C. et al. Regional brain activation changes and abnormal functional connectivity of the ventrolateral prefrontal cortex during working memory processing in adults with attention-deficit/hyperactivity disorder. Hum Brain Mapp 30, 2252-2266 (2009).

26. Wang, X., Jiao, Y., Tang, T., Wang, H. \& Lu, Z. Altered regional homogeneity patterns in adults with attention-deficit hyperactivity disorder. Eur J Radiol 82 1552-1557 (2013).

27. Shaw, P. et al. Attention-deficit/hyperactivity disorder is characterized by a delay in cortical maturation. Proc Natl Acad Sci U S A 104, 19649-19654 (2007).

28. Sowell, E. R. et al. Cortical abnormalities in children and adolescents with attention-deficit hyperactivity disorder. Lancet 362, 1699-1707 (2003)

29. Kobel, M. et al. Structural and functional imaging approaches in attention deficit/ hyperactivity disorder: does the temporal lobe play a key role? Psychiatry Res 183, 230-236 (2010).

30. Spinelli, S. et al. Variability in post-error behavioral adjustment is associated with functional abnormalities in the temporal cortex in children with ADHD. J Child Psychol Psychiatry 52, 808-816 (2011).

31. Dibbets, P., Evers, E. A., Hurks, P. P., Bakker, K. \& Jolles, J. Differential brain activation patterns in adult attention-deficit hyperactivity disorder (ADHD) associated with task switching. Neuropsychology 24, 413-423 (2010).

32. Vance, A. et al. Right parietal dysfunction in children with attention deficit hyperactivity disorder, combined type: a functional MRI study. Mol Psychiatry 12 , 826-832, 793 (2007).

33. Bonelli, R. M. \& Cummings, J. L. Frontal-subcortical circuitry and behavior. Dialogues Clin Neurosci 9, 141-151 (2007).

34. Nagel, B. J. et al. Altered white matter microstructure in children with attentiondeficit/hyperactivity disorder. J Am Acad Child Adolesc Psychiatry 50, 283-292 (2011).

35. Soliva, J. C. Neuroimaging in the diagnosis of ADHD: where we are and where we are going. Expert Opin Med Diagn 5, 307-318 (2011).

36. Xia, S. et al. Thalamic shape and connectivity abnormalities in children with attention-deficit/hyperactivity disorder. Psychiatry Res 204, 161-167 (2012).

37. Duerden, E. G., Tannock, R. \& Dockstader, C. Altered cortical morphology in sensorimotor processing regions in adolescents and adults with attention-deficit/ hyperactivity disorder. Brain Res 1445, 82-91 (2012).

38. Sripada, C. et al. Disrupted network architecture of the resting brain in attentiondeficit/hyperactivity disorder. Hum Brain Mapp 35, 4693-4705 (2014).
39. Jonh Kaas \& Stepniewska, I. Motor cortex. Encyclopedia of the Human Brain Volume 3 (eds. Vilayanur Ramachandran), 159-169, (Elsevier Science, San Diego, 2002).

40. Aron, A. R. From reactive to proactive and selective control: developing a richer model for stopping inappropriate responses. Biol Psychiatry 69, e55-68 (2011).

41. Lei, D. et al. Altered brain activation during response inhibition in children with primary nocturnal enuresis: an fMRI study. Hum Brain Mapp 33, 2913-2919 (2012).

42. Cortese, S. \& Castellanos, F. X. Neuroimaging of attention-deficit/hyperactivity disorder: current neuroscience-informed perspectives for clinicians. Curr Psychiatry Rep 14, 568-578 (2012).

43. Roth, R. M. \& Saykin, A. J. Executive dysfunction in attention-deficit/ hyperactivity disorder: cognitive and neuroimaging findings. Psychiatr Clin North Am 27, 83-96, ix (2004).

44. Sun, S. W., Liang, H. F., Schmidt, R. E., Cross, A. H. \& Song, S. K. Selective vulnerability of cerebral white matter in a murine model of multiple sclerosis detected using diffusion tensor imaging. Neurobiol Dis 28, 30-38 (2007).

45. Suzuki, Y., Matsuzawa, H., Kwee, I. L. \& Nakada, T. Absolute eigenvalue diffusion tensor analysis for human brain maturation. NMR Biomed 16, 257-260 (2003).

46. Gau, S. S. et al. Psychometric properties of the Chinese version of the Swanson, Nolan, and Pelham, version IV scale - parent form. Int J Methods Psychiatr Res 17, 35-44 (2008).

47. Swanson, J. M. et al. Clinical relevance of the primary findings of the MTA: success rates based on severity of ADHD and ODD symptoms at the end of treatment. J Am Acad Child Adolesc Psychiatry 40, 168-179 (2001).

48. Lei, D. et al. Changes in the brain microstructure of children with primary monosymptomatic nocturnal enuresis: a diffusion tensor imaging study. PLoS One 7, e31023 (2012)

49. Ashburner, J. \& Friston, K. J. Voxel-based morphometry--the methods. Neuroimage 11, 805-821 (2000).

50. Ledberg, A., Akerman, S. \& Roland, P. E. Estimation of the probabilities of 3D clusters in functional brain images. Neuroimage 8, 113-128 (1998).

\section{Acknowledgments}

This research was supported by grants from the National Natural Science Foundation of China (Grant Nos. 81030027 and 81201082), the Shanghai Children's Medical Center Fund (Grant No. 10DZ2272200), the Shanghai Key Laboratory of Children's Environmental Health (Grant No09DZ2200900), the Development of Science and Technology Fund of Pudong New Area, Shanghai (Grant No. PKJ2009-Y03), and the China Postdoctoral Science Foundation (Grant Nos. 2012M521696 and 2013T60856).

\section{Author contributions}

X.D. and Q.G. conceptualized the project. D.L. and X.D. designed the protocol and wrote the main manuscript text. D.L., J.M., X.D., X.J. and G.S. performed the experiments. D.L. and X.D. conducted the statistical analyses. All authors reviewed the manuscript. D.L. and Q.G. revised the manuscript.

\section{Additional information}

Competing financial interests: The authors declare no competing financial interests.

How to cite this article: Lei, D. et al. Microstructural Abnormalities in the Combined and Inattentive Subtypes of Attention Deficit Hyperactivity Disorder: a Diffusion Tensor Imaging Study. Sci. Rep. 4, 6875; DOI:10.1038/srep06875 (2014)

This work is licensed under a Creative Commons Attribution-NonCommercialNoDerivs 4.0 International License. The images or other third party material in this article are included in the article's Creative Commons license, unless indicated otherwise in the credit line; if the material is not included under the Creative Commons license, users will need to obtain permission from the license holder in order to reproduce the material. To view a copy of this license, visit http:// creativecommons.org/licenses/by-nc-nd/4.0/ 